environment ${ }^{8}$, and it promotes the overgrowth of polarity-deficient cells when they are surrounded by wild-type cells ${ }^{9}$. Although these results caution against oversimplification, it is tempting to speculate that changes in cell fitness and cell behavior induce changes in cell identity and that epithelial cells have an intrinsic ability to recognize whether their neighbors are different via a variety of immune-related pathways. There are, without a doubt, many fascinating questions to answer, but three immediately spring to mind. How do fitness and behavior differences activate immune-related molecules? Did the roles of the immune pathways in epithelial cells arise before the evolution of the immune cells in which these pathways are best known to act? And how do oncogenic cells manage to evade these surveillance mechanisms? Answering these question will hopefully not only provide important insight into how tissue fitness is maintained, but also incite new therapeutic options for diseases such as cancer.

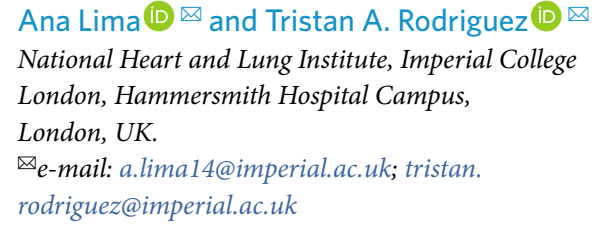
National Heart and Lung Institute, Imperial College London, Hammersmith Hospital Campus, London, UK.

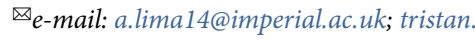
rodriguez@imperial.ac.uk

Published online: 22 October 2021

https://doi.org/10.1038/s41590-021-01053-6

References

1. Ayakawa, S. et al. Nat. Immunol. https://doi.org/10.1038/s41590021-01045-6 (2021).

2. Kon, S. \& Fujita, Y. Dev. Biol. 476, 112-116 (2021).

3. Bowling, S., Lawlor, K. \& Rodriguez, T. A. Development 146, dev167486 (2019)

4. Kajita, M. et al. Nat. Commun. 5, 4428 (2014).

5. Franceschi, R. T. \& Xiao, G. J. Cell. Biochem. 88, 446-454 (2003).

6. Takai, T. Immunology 115, 433-440 (2005).

7. Meyer, S. N. et al. Science 346, 1258236 (2014).

8. Germani, F., Hain, D., Sternlicht, D., Moreno, E. \& Basler, K. eLife 7, e39939 (2018).

9. Katsukawa, M., Ohsawa, S., Zhang, L., Yan, Y. \& Igaki, T. Curr. Biol. 28, 1756-1767.e1756 (2018).

Competing interests

The authors declare no competing interests.

\title{
ISG15 secretion exacerbates inflammation in SARS-CoV-2 infection
}

\begin{abstract}
Conjugation of the ubiquitin-like protein ISG15 to targets (ISGylation) benefits antiviral defense. However, SARS-CoV-2 induces human macrophages to preferentially secrete ISG15 via its papain-like protease, and extracellular non-conjugated ISG15 acts as a cytokine to exacerbate SARS-CoV-2-triggered inflammation.
\end{abstract}

\section{Xuetao Cao}

$\mathrm{V}$ iral immunoevasion, excessive inflammation and cytokine storm are associated with the severity and the mortality of coronavirus disease 2019 (COVID-19) caused by infection with the severe acute respiratory syndrome (SARS) coronavirus (CoV) 2 (SARS-CoV-2). Some potential immunotherapeutic strategies have been clinically tested to control the progression of COVID-19, such as blockade of proinflammatory cytokine production and function. Efficient activation of an innate immune response is required for elimination of invading viruses, but its aberrant activation and overproduction of proinflammatory cytokines may induce host tissue damage. An imbalanced innate immune response has been reported to contribute to the immunopathology of COVID-19, which is characterized by a weak type I interferon (IFN-I, such as IFN- $\alpha$ and IFN- $\beta$ ) response and exacerbated release of proinflammatory cytokines (including IL-1 $\beta$, IL-6 and TNF, along with a mixture of chemokines, including CCL2, CCL3 and CXCL8) ${ }^{1,2}$. On the basis of investigations of evasion mechanisms imposed by SARS-CoV-2-encoded proteins, it is partially understood why the IFN-I production and IFN-I-induced antiviral effects are impaired ${ }^{1}$. However, it remains mysterious as to why the proinflammatory cytokine response is predominantly exacerbated by SARS-CoV-2 infection. In this issue of Nature Immunology, Sanyal and colleagues show that SARS-CoV-2 infection of human macrophages induces the release of intracellular ISG15 (interferon-stimulated gene 15 , one of the targets of the IFN-I response) through the viral papain-like protease (PLpro), and then the extracellular free ISG15 acts in a cytokine-like manner to exaggerate the subsequent secretion of multiple proinflammatory cytokines and chemokines (Fig. 1), which potentially contributes to the clinical super-inflammatory response of COVID-19 (ref. ${ }^{3}$ ). The findings by Sanyal and colleagues not only provide mechanistic insights into the immunopathogenesis of the exacerbated inflammation triggered by SARS-CoV-2 but also imply that either blockade of the virus' PLpro activity or neutralization of extracellular ISG15 are potential strategies for handling super-inflammation in patients with COVID-19.

Upon recognition of viral RNAs, the RNA sensors such as RIG-I and MDA5 can initiate downstream innate signaling by activating the kinases TBK1 and IKK $\varepsilon$ as well as the transcription factors IRF3 and IRF7, leading to gene expression of IFN-I and proinflammatory cytokines. IFN-I molecules activate a JAK-STAT pathway to induce hundreds of ISGs, including ISG15, to directly or indirectly establish antiviral innate effects ${ }^{4}$. RIG-I and/or MDA5 have been implicated as potential SARS-CoV-2 sensors, and SARS-CoV-2 can evade antiviral innate responses by disrupting ISG15-dependent MDA5 activation $^{5,6}$. Together with the identification of extracellular free ISG15 as a cytokine to induce innate inflammation upon pathogen infection $^{4,7}$, the findings by Sanyal and colleagues suggest a mode of second-wave innate inflammation that is triggered and amplified by SARS-CoV-2-mediated release of extracellular ISG15.

ISG15, a $15 \mathrm{kDa}$ ubiquitin-like and interferon-inducible protein, has been 


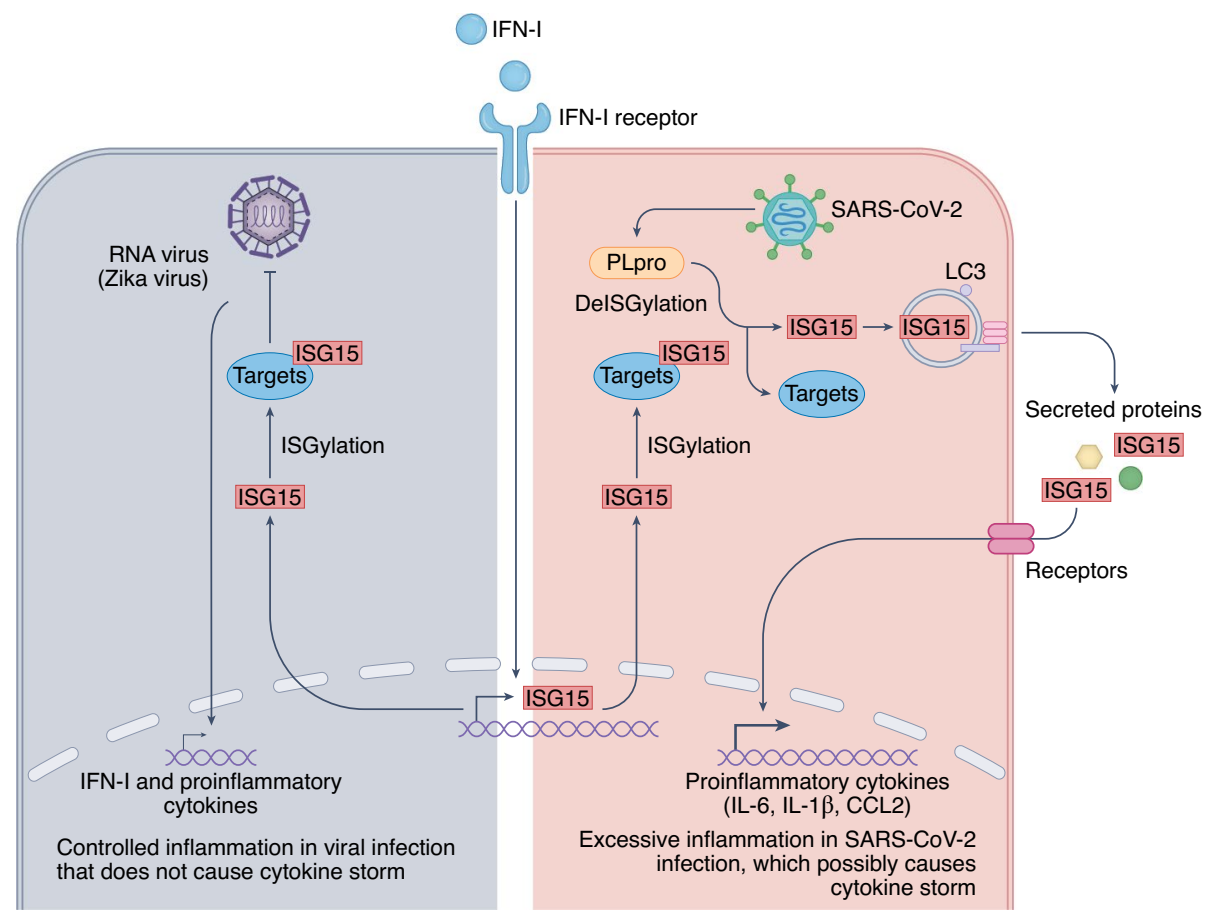

Fig. 1 | Preferentially secreted, non-conjugated ISG15 feedback stimulates macrophages to exacerbate inflammatory response triggered by SARS-CoV-2 infection. In response to viral infection, host cells initiate innate signaling to induce the expression of IFN-I and proinflammatory cytokines. IFN-I molecules induce hundreds of ISGs, including ISG15, to elicit antiviral innate response. Conjugation of ISG15 to intracellular target proteins, which is known as ISGylation, contributes to the defense against viruses. However, SARS-CoV-2 infection induces a weak IFN-I response and an exacerbated release of proinflammatory cytokines (including IL-6 and IL-1 $\beta$ ) and chemokines (including CCL2), which are associated with the immunopathology of COVID-19. SARS-CoV-2 can motivate macrophages to preferentially secrete ISG15 via its PLpro (as a deISGylation enzyme), and in turn, the extracellular free ISG15 acts as cytokine to amplify macrophage production of proinflammatory cytokines and chemokines, which thus exacerbates SARS-CoV-2-triggered inflammation and possibly causes cytokine storm in COVID-19. LC3, microtubule-associated protein 1 light chain 3.

regarded as a kind of moiety conjugatable to protein substrates and implicated in the defense against many viral pathogens ${ }^{4}$. Conjugation of ISG15, commonly known as ISGylation, is a three-step enzymatic cascade: the E1 enzyme UBE1L (also known as UBA7), the E2 enzyme UBCH8 (also known as UBE2L6) and the predominant E3 enzyme HERC5, whereas USP18 (also known as UBP43) mediates the deISGylation process. The supporting evidence for ISG15 in antiviral responses mostly relies on direct ISGylation of diverse viral proteins by ISG15, which results in the inhibition of viral replication as well as the interruption of virus life cycle $^{4}$. Controversies exist as to the role of ISG15 in antiviral responses, for example in the requirement of ISG15 for antiviral responses in mouse but not human cells ${ }^{4,8,9}$. ISG15-deficient patients even show an enhanced IFN-I signature, possibly due to the decreased stability of
USP18 (a negative regulator of IFNAR signaling $)^{10}$. In host cells, ISG15 exists in three forms: conjugated ISG15 in ISGylated proteins, free intracellular ISG15 that can non-covalently bind to client proteins and secreted extracellular ISG15. ISGylation of MDA5 triggers activation of innate immunity, ISGylation of IRF3 sustains its activation, and ISGylation of STAT1 preserves its phosphorylation and activation ${ }^{4,6,11}$. In addition, ISG15 non-covalently interacts with USP18 in human cells but inhibits its degradation. Intracellular ISG15 binds to the histone deacetylase HDAC6 and RIG-I as well as ubiquitin-binding protein p62 to regulate autophagic clearance of HDAC6 and RIG-I ${ }^{4,10}$. When existing as an extracellular free factor, ISG15 has been proposed to function as a cytokine by mediating signaling following binding to the cell surface integrin receptor CD11a-CD18 (ref. $\left.{ }^{4}\right)$. These findings indicate that the multiple functions of ISG15 in innate immunity and inflammation depend on its targets and partners in different locations.

Sanyal and colleagues showed that infection of human peripheral blood monocyte-derived macrophages by SARS-CoV-2 induced expression of ISGylation enzymes, including ISG15, UBE1L, UBCH8 and HERC5, as well as USP18. However, SARS-CoV-2 did not induce robust ISGylation of cellular proteins but instead promoted the release of ISG15.

This was not due to IFN-I but was caused by live viral infection. The significant secretion of free ISG15 by macrophages was selectively triggered by SARS-CoV-2, and neither Zika nor influenza viruses could do this. ISG15 release occurred in an autophagy-dependent manner and by an unconventional secretory mechanism. These findings are consistent with previous reports showing that ISGylation machinery is IFN-I-inducible and ISG15 is released upon pathogen infection ${ }^{4,7}$. Intracellular ISGylation might serve as an inhibitory mechanism for the secretion of inflammatory factors (such as IL-1 $\beta$, CCL2 and IL-6), as Sanyal and colleagues found that knockdown of ISG15, UBE1L and HERC5 reduced macrophage production of these cytokines upon SARS-CoV-2 infection. Therefore, the authors propose that a higher ratio of free versus conjugated ISG15 in macrophages could correlate with polarization of inflammatory M1 macrophages and be an indicator for the severity of SARS-CoV-2mediated inflammation ${ }^{3}$. Supporting evidence was raised from the authors' quantitative proteomic assays of intracellular ISGylated proteins and the secretome of ISG15-knockout or -knockdown cells. Within the ISGylome protein profiles, proteins, such as RIG-I, STAT1-3, UBE1L, UBCH8 and HERC5, which have been previously reported to be co-operators, targets or partners of ISG $15^{4}$, were downregulated in ISG15-knockout cells. The most convincing evidence for secreted ISG15 as an amplifier of inflammation was from Sanyal and colleagues' experiments using recombinant ISG15 to stimulate ISG15-knockdown cells to release inflammatory cytokines ${ }^{3}$.

The clinical significance of ISG15 secretion in SARS-CoV-2 infection of macrophages is still uncertain, even though Sanyal and colleagues could observe elevated ISG15 in the serum of patients with COVID-19 at 1 week of disease onset ${ }^{3}$. Further analysis of the correlation of serum ISG15 levels to the other inflammatory cytokines, clinical manifestations and pathogenesis at different stages of COVID19 in larger cohorts would be necessary 
to substantiate its significance. Further investigations using recombinant ISG15 may help to understand the secretome affected by extracellular ISG15 and the mechanisms for underlying the ISG15-triggered secretion pathway in macrophages and possibly in tissue-resident and migratory macrophages.

Another exciting observation by Sanyal and colleagues is the confirmation of the PLpro encoded by SARS-CoV-2 as a deISGylation enzyme for immunoevasion and inflammatory cytokine production ${ }^{3}$. PLpro is one of the non-structural proteins (Nsp3) encoded by the SARS-CoV-2 genome that acts as an enzyme to cleave proteins or remove ubiquitin or ISG15 from modified proteins. PLpro is required for the efficient cleavage of viral polyprotein, a process that is essential for viral genome transcription and replication. SARS-CoV-2 PLpro could enzymatically decrease the ISGylation of MDA5 and IRF3 to inhibit antiviral innate responses ${ }^{6,11}$. Sanyal and colleagues revealed that PLpro, but not catalytically inactive PLpro, promoted the release of proinflammatory cytokines and ISG15 in SARS-CoV-2-infected cells ${ }^{3}$, which thus highlights PLpro as an attractive candidate to be targeted to reverse the subverted antiviral innate response and attenuate the exacerbated inflammatory storm. Future work may address how PLpro or ISG15 affect the autophagy-related secretion pathway, what is a realistic method to selectively block cellular release of free ISG15 in response to SARS-CoV-2 infection, and what the effects of PLpro inhibitors on the SARS-CoV-2-induced inflammatory cytokine storm and lung damage are.

The article by Sanyal and colleagues reveals an important role of extracellular ISG15 in amplifying SARS-CoV-2-induced inflammation and proposes PLpro as a critical target to reverse viral evasion and counteract SARS-CoV-2-induced super-inflammation. Whether neutralization of free ISG15 can be realized clinically and be also applicable to the control of other virus-induced inflammatory diseases are questions worthy of further exploration.
Xuetao Cao (iD) 1,2四

${ }^{1}$ College of Life Sciences, Nankai University,

Tianjin, China. ${ }^{2}$ Department of Immunology,

Institute of Basic Medical Sciences, Chinese Academy

of Medical Sciences, Beijing, China.

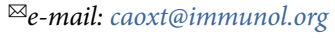

Published online: 20 October 2021

https://doi.org/10.1038/s41590-021-01056-3

References

1. Lowery, S. A., Sariol, A. \& Perlman, S. Cell Host Microbe 29 , 1052-1062 (2021).

2. Cao, X. Nat. Rev. Immunol. 20, 269-270 (2020).

3. Munnur, D. et al. Nat. Immunol. https://doi.org/10.1038/s41590021-01035-8 (2021)

4. Perng, Y. C. \& Lenschow, D. J. Nat. Rev. Microbiol. 16, 423-439 (2018)

5. Yamada, T. et al. Nat. Immunol. 22, 820-828 (2021).

6. Liu, G. et al. Nat. Microbiol. 6, 467-478 (2021)

7. Swaim, C. D. et al. Cell Rep. 31, 107772 (2020).

8. Lenschow, D. J. et al. Proc. Natl Acad. Sci. USA 104, 1371-1376 (2007).

9. Speer, S. D. et al. Nat. Commun. 7, 11496 (2016).

10. Zhang, X. et al. Nature 517, 89-93 (2015).

11. Shin, D. et al. Nature 587, 657-662 (2020).

Competing interests

The author declares no competing interests. 